\title{
Hysteresis in quantized vortex shedding
}

\author{
Tsuyoshi Kadokura, ${ }^{1}$ Jun Yoshida, ${ }^{1}$ and Hiroki Saito ${ }^{1}$ \\ ${ }^{1}$ Department of Engineering Science, University of Electro-Communications, Tokyo 182-8585, Japan
}

(Dated: July 7, 2021)

\begin{abstract}
It is shown using numerical simulations that flow patterns around an obstacle potential moving in a superfluid exhibit hysteresis. In a certain velocity region, there is a bistability between stationary laminar flow and periodic vortex shedding. The bistability exists in two and three dimensional systems.

PACS numbers: 03.75.Lm, 47.32.ck, 47.37.+q, 67.85.De
\end{abstract}

\section{INTRODUCTION}

The dynamics of fluids can exhibit hysteresis. For example, a flag-like object shows bistability between flapping and nonflapping states [1, 2]. Hysteresis also exists in vortex shedding dynamics behind rigid objects, such as a vibrating cylinder [3], a multiple cylinder arrangement [4], a long cylinder in a three-dimensional flow [5], and a rod in a soap film [6]. In these experiments, the transitions between laminar flow and vortex shedding states occur in a hysteretic manner as a function of the Reynolds number. It is known that the Taylor-Couette flow also exhibits hysteresis [7]. In superfluids, hysteresis has been observed in rotating toroidal systems [8, [9].

In this paper, we consider the transition between a laminar flow state and a quantized vortex shedding state around an obstacle moving in a Bose-Einstein condensate (BEC). In a superfluid, the velocity field around an obstacle is irrotational below the critical velocity. When the velocity of the obstacle exceeds the critical velocity, quantized vortices are created and released behind the obstacle, as observed in a trapped BEC stirred by an optical potential [10 12]. The critical velocity for vortex creation and the dynamics of quantized vortex shedding in superfluids have been studied theoretically by many researchers $[13$ 27].

The purpose of the present paper is to show that superfluids undergo hysteretic changes between stationary laminar flow and periodic shedding of quantized vortices. Consider an obstacle with gradually increasing velocity; on reaching the critical velocity $V_{c 1}$, periodic vortex shedding starts. Now consider an obstacle with gradually decreasing velocity from above $V_{c 1}$; the vortex shedding stops at a velocity $V_{c 2}$. We show that there is a bistability between these flow patterns, i.e., $V_{c 2}<V_{c 1}$. Although hysteretic vortex shedding under a moving potential was reported in Ref. [24], the mechanism has not been studied in detail. In the present paper, we show that the hysteretic behaviors are due to the fact that released vortices enhance the flow velocity around the obstacle and induce subsequent vortex creation. We show that the hysteretic behavior is observed for a circular obstacle moving in a two-dimensional (2D) superfluid and a spherical obstacle moving in a three-dimensional (3D) superfluid.

This paper is organized as follows. Section II formu- lates the problem and describes the numerical method. The hysteretic dynamics are studied for a 2D system in Sec. IIIA and for a 3D system in Sec. IIIB Conclusions are given in Sec. IV.

\section{FORMULATION OF THE PROBLEM}

We study the dynamics of a BEC at zero temperature using mean-field theory. The system is described by the Gross-Pitaevskii (GP) equation,

$$
i \hbar \frac{\partial \Psi}{\partial t}=-\frac{\hbar^{2}}{2 m} \nabla^{2} \Psi+U(\boldsymbol{r}, t) \psi+\frac{4 \pi \hbar^{2} a}{m}|\Psi|^{2} \Psi
$$

where $\Psi(\boldsymbol{r}, t)$ is the macroscopic wave function, $m$ is the atomic mass, $U(\boldsymbol{r}, t)$ is an external potential, and $a$ is the $s$-wave scattering length. We consider situations in which a localized potential $u$ moves at a velocity $\boldsymbol{V}(t)$, i.e., the potential $U$ has a form,

$$
U(\boldsymbol{r}, t)=u\left(\boldsymbol{r}-\int^{t} \boldsymbol{V}\left(t^{\prime}\right) d t^{\prime}, t\right) .
$$

We transform Eq. (11) into the frame of reference of the moving potential $U$ by substituting the unitary transformation

$$
\Psi(\boldsymbol{r}, t)=\exp \left[-\int^{t} \boldsymbol{V}\left(t^{\prime}\right) d t^{\prime} \cdot \nabla\right] \psi(\boldsymbol{r}, t)
$$

into Eq. (11), which yields

$i \hbar \frac{\partial \psi}{\partial t}=-\frac{\hbar^{2}}{2 m} \nabla^{2} \psi+i \hbar \boldsymbol{V} \cdot \boldsymbol{\nabla} \psi+u(\boldsymbol{r}, t) \psi+\frac{4 \pi \hbar^{2} a}{m}|\psi|^{2} \psi$.

In the following, the velocity vector is taken as

$$
\boldsymbol{V}(t)=-V(t) \hat{x},
$$

where $\hat{x}$ is the unit vector in the $x$ direction.

We consider an infinite system, in which the atomic density $|\psi|^{2}$ far from the moving potential is constant $n_{0}$. For the density $n_{0}$, the healing length $\xi$ and the sound velocity $v_{s}$ are defined as

$$
\xi=\frac{1}{\sqrt{4 \pi n_{0} a}}, \quad v_{s}=\frac{\hbar \sqrt{4 \pi n_{0} a}}{m},
$$


which determine the characteristic time scale,

$$
\tau=\frac{\xi}{v_{s}}
$$

The chemical potential for the density $n_{0}$ is given by

$$
\mu=\frac{4 \pi \hbar^{2} a}{m} n_{0} .
$$

Normalizing Eq. (4) by the quantities in Eqs. (6) $-(8)$, we obtain

$$
i \frac{\partial \tilde{\psi}}{\partial \tilde{t}}=-\frac{1}{2} \tilde{\nabla}^{2} \tilde{\psi}+i \tilde{\boldsymbol{V}} \cdot \tilde{\nabla} \tilde{\psi}+\tilde{u} \tilde{\psi}+|\tilde{\psi}|^{2} \tilde{\psi}
$$

where $\tilde{\psi}=n_{0}^{-1 / 2} \psi, \tilde{t}=t / \tau, \tilde{\nabla}=\xi \nabla, \tilde{\boldsymbol{V}}=\boldsymbol{V} / v_{s}$, and $\tilde{u}=u / \mu$ are dimensionless quantities. The independent parameters in Eq. (9) are only $\tilde{\boldsymbol{V}}$ and $\tilde{u}$.

We numerically solve Eq. (9) using the pseudo-spectral method [28]. The initial state is the stationary state of Eq. (9) for a velocity $V$ below the critical velocity $V_{c 1}$ for vortex nucleation, which is prepared by the imaginarytime propagation method [29]. The initial state is a stationary laminar flow and contains no vortices. To break the exact numerical symmetry, a small random noise is added to each mesh of the initial state. The real-time propagation of Eq. (9) is then calculated with a change in the velocity $V$ or the potential $u$ to trigger the vortex creation. The size of the space is taken to be large enough and the periodic boundary condition imposed by the pseudo-spectral method does not affect the dynamics around the potential.

\section{NUMERICAL RESULTS}

\section{A. Two dimensional system}

First, we consider a 2D space. Typically, the size of the numerical space is taken to be $512 \xi$ in $x$ and $256 \xi$ in $y$, and is divided into a $8192 \times 4096$ mesh. The obstacle potential is given by

$$
u(\boldsymbol{r})= \begin{cases}\infty & \left(\sqrt{x^{2}+y^{2}}<R\right), \\ 0 & \left(\sqrt{x^{2}+y^{2}}>R\right),\end{cases}
$$

where $R$ is the radius of the circular potential. Numerically, a value that is significantly larger than the chemical potential is used for $\infty$ in Eq. (10). The following results are qualitatively the same as those for a Gaussian potential in place of the rigid circular potential in Eq. (10).

Figure 1 shows the time evolution of the density $|\psi|^{2}$ and phase $\arg \psi$ profiles. The initial state is the stationary state for the velocity $v=0.43 v_{s}$ and radius $R=4.1 \xi$, as shown in Fig. 1(a). This stationary laminar flow state is stable. To trigger the vortex shedding, we apply an additional potential,

$$
u_{\text {add }}(\boldsymbol{r})=\mu e^{-\left[x^{2}+(y-R)^{2}\right] / \xi^{2}}
$$
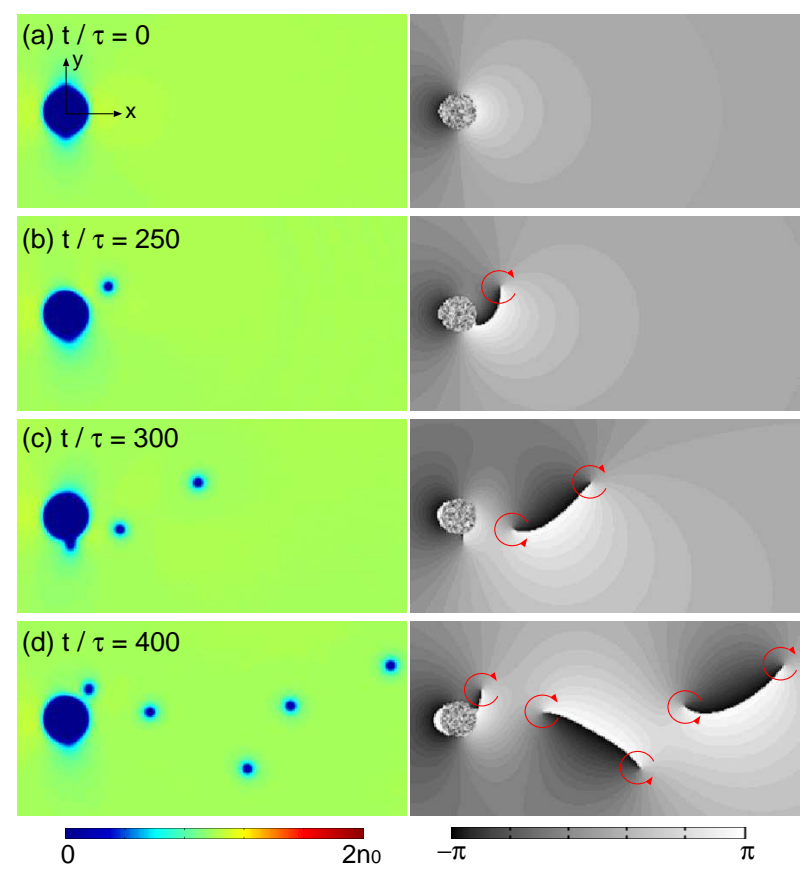

FIG. 1: (color online) Time evolution of the density $|\psi|^{2}$ (left panels) and phase $\arg \psi$ (right panels) profiles for $V=0.43 v_{s}$ and $R=4.1 \xi$. To trigger the vortex shedding, the additional potential given by Eq. (11) is applied during $200<t / \tau<220$. The arrows in the phase profiles indicate the directions in which the quantized vortices are rotating. The size of each panel is $80 \xi \times 40 \xi$. See the Supplemental Material for a movie of the dynamics.

during $200<t / \tau<220$, in addition to the circular potential in Eq. (10). This additional potential perturbs the edge of the circular potential, at which quantized vortex creation is induced, as shown in Fig. 1(b). Subsequently, quantized vortices are periodically created one after the other [23], as shown in Figs. 1(c) and1(d), even after the perturbation potential is removed at $t=220 \tau$ and the velocity $v=0.43 v_{s}$ is smaller than the critical velocity $V_{c 1}$. This result indicates that there are at least two stable flow patterns for the same parameters: a stationary laminar flow and periodic vortex shedding.

The velocity field of the atomic flow has the form,

$$
\boldsymbol{v}(\boldsymbol{r}, t)=\frac{\hbar}{2 m i|\psi|^{2}}\left(\psi \boldsymbol{\nabla} \psi^{*}-\psi^{*} \nabla \psi\right)-\boldsymbol{V} .
$$

Figure 2(a) shows the time evolution of the velocities $v_{x}$ at $(x,|y|)=(0, R+2 \xi)$. These positions are indicated by the crosses in Fig. 2(b). For the stationary flow $(t<200 \tau)$, the velocities are $v_{x} \simeq v_{s}$. The fluctuations around $v_{s}$ are due to the small numerical noises added to the initial state. At $t=200 \tau$, the additional potential given by Eq. (11) is applied and a clockwise vortex is released from near the position $(0, R)$. As a consequence, $v_{x}(0, R+2 \xi)$ suddenly decreases. It can also be seen in Fig. 2(c) that the released vortex decreases the velocity field in the vicinity of its creation. The clockwise vortex 

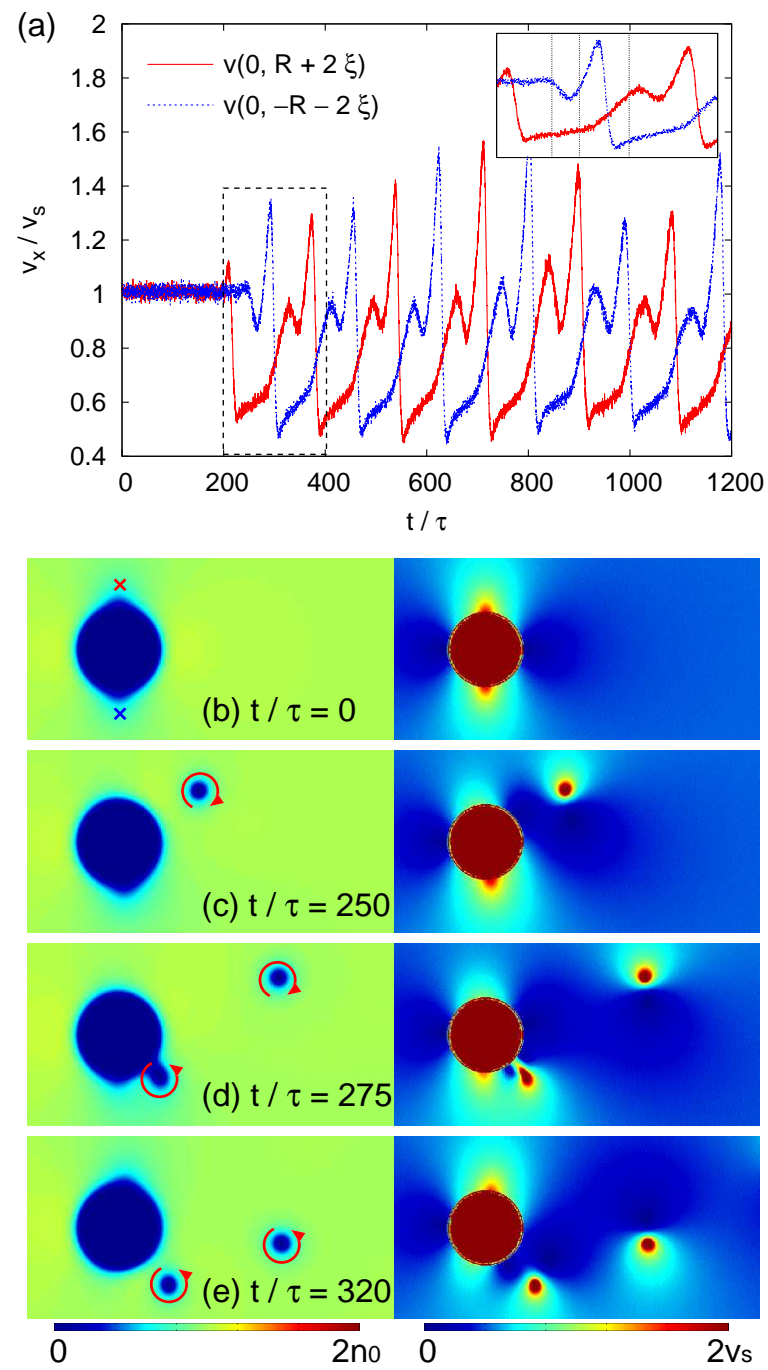

FIG. 2: (color online) (a) Time evolution of the velocity $v_{x}$ at $(x, y)=(0, \pm R \pm 2 \xi)$ for the same parameters as those in Fig. 11 The dashed square is magnified in the inset, where the vertical lines indicate $t / \tau=250,275$, and 320 . (b)(e) Density $|\psi|^{2}$ profiles (left panels) and velocity $|\boldsymbol{v}|$ profiles (right panels). The crosses in (b) indicate the positions $(x,|y|)=(0, R+2 \xi)$ at which the velocities are plotted in (a). The arrows in the density profiles indicate the directions in which the quantized vortices are rotating. The size of each panel is $40 \xi \times 20 \xi$.

shedding then induces counterclockwise vortex creation, as shown in Fig. 2(d). Immediately after that $(t=275 \tau$ $320 \tau), v_{x}(0,-R-2 \xi)$ increases rapidly, which is followed by a sudden decrease due to the shedding of another counterclockwise vortex, as shown in Fig. 2(d). This periodic vortex shedding is repeated indefinitely. The dynamics shown in Fig. 2 implies that the release of a vortex induces the creation of a subsequent vortex, i.e., periodic vortex shedding is taking place.

To show the hysteresis clearly, we gradually increase and decrease the velocity $V(t)$ around the critical veloc-

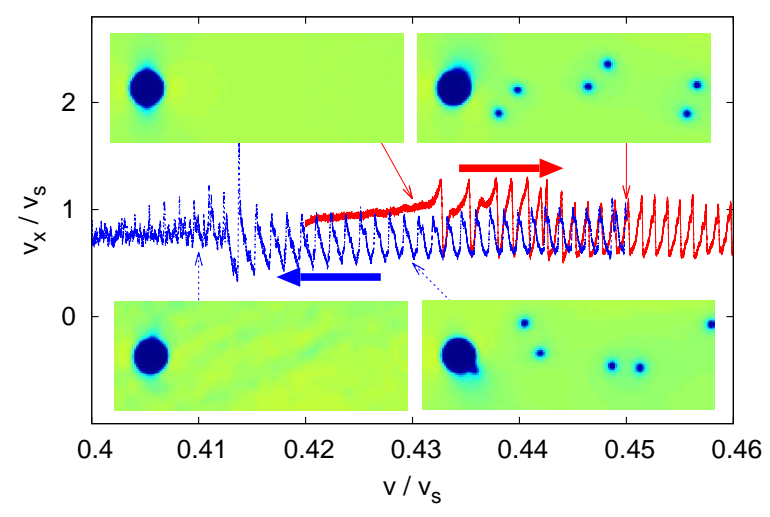

FIG. 3: (color online) Time evolution of $v_{x}$ at $(x, y)=(0, R+$ $2 \xi$ ) for $R=4.1 \xi$. The velocity is increased as $V(t) / v_{s}=$ $0.42+10^{-5} t / \tau$ (red, solid line) or decreased as $V(t) / v_{s}=$ $0.45-10^{-5} t / \tau$ (blue, dashed line). The insets show the density profiles at $V(t) / v_{s}=0.43$ and 0.45 for the increase in $V(t)$, and 0.43 and 0.41 for the decrease in $V(t)$.

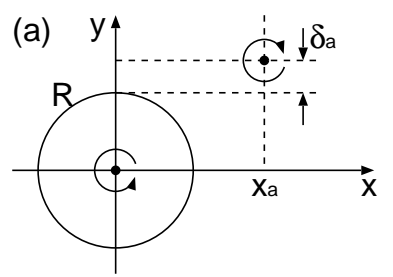

(b)

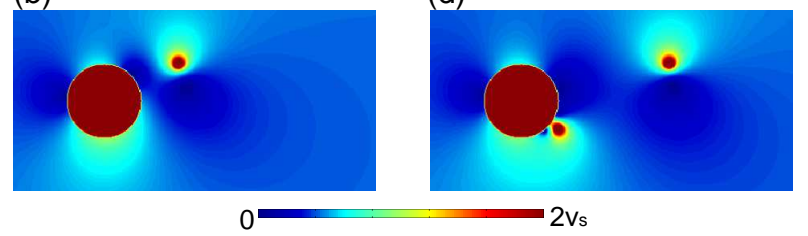

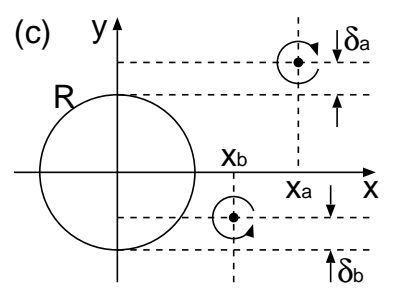

(d)
FIG. 4: (color online) (a) and (c) Schematic illustrations of the point-vortex model used to derive Eqs. (13) and (15), respectively. (b) $|\boldsymbol{v}|$ in Eq. (13) for $x_{a}=2 R$ and $\delta_{a}=0$. (d) $|\boldsymbol{v}|$ in Eq. (15) for $x_{a}=4 R, x_{b}=R, \delta_{a}=0$, and $\delta_{b}=\xi$. The size of each panel in (b) and (d) is $40 \xi \times 20 \xi$.

ity. Figure 3 shows the time evolution of the flow velocity $v_{x}$ at $(x, y)=(0, R+2 \xi)$. When the velocity $V(t)$ is gradually increased, the vortex shedding starts at the critical velocity $V_{c 1} \simeq 0.432 v_{s}$. On the other hand, when $V(t)$ is decreased from above $V_{c 1}$, the periodic vortex shedding continues for $V(t)<V_{c 1}$, eventually stopping at the lower critical velocity $V_{c 2} \simeq 0.412 v_{s}$. The fluctuation in $v_{x}$ for $V(t) \lesssim 0.41 v_{s}$ is due to the remnant disturbing waves.

The velocity field around a circular obstacle can be analyzed using the point-vortex model for an inviscid incompressible fluid. The situation in Fig. 2(c) is modeled as in Fig. 4(a), where a clockwise vortex is located at $(x, y)=\left(x_{a}, R+\delta_{a}\right)$ and the circle of radius $R$ contains a counterclockwise vortex. The complex velocity field in 
which the normal component $\boldsymbol{v} \cdot \boldsymbol{r}$ vanishes at $r=R$ is given by

$$
v_{x}-i v_{y}=V\left(1-\frac{R^{2}}{z^{2}}\right)+\frac{\Gamma}{2 \pi i}\left(-\frac{1}{z-z_{a}}+\frac{1}{z-z_{a}^{\prime}}\right),
$$

where $z=x+i y, \Gamma=h / m, z_{a}=x_{a}+i\left(R+\delta_{a}\right)$, and $z_{a}^{\prime}=R^{2} / z_{a}^{*}$ [30]. The first term on the right-hand side of Eq. (13) approaches a uniform flow $\left(v_{x}, v_{y}\right)=(V, 0)$ at infinity $|z| \rightarrow \infty$ and the second term represents a flow generated by the vortices located at $z_{a}$ and the origin. The flow velocity at $z= \pm i R$ is

$$
v_{x}=2 V \mp \frac{\Gamma}{2 \pi} \frac{x_{a}^{2}+\delta_{a}\left(2 R+\delta_{a}\right)}{x_{a}^{2}+\left(R \mp R+\delta_{a}\right)^{2}}
$$

and $v_{y}=0$, which indicates that the flow velocity at $z \simeq-i R$ is enhanced by the vortices. Thus, once a vortex is released from $z \simeq i R$, the next vortex is created at $z \simeq$ $-i R$, which results in the dynamics shown in Fig. 2 (d). The velocity field in Eq. (13) for $x_{a}=2 R$ and $\delta_{a}=0$ is shown in Fig. 4(b), which is very similar to Fig. 2(c).

The situation in Fig. 2(d) is modeled by Fig. 4(c), for which the velocity field is given by

$$
\begin{aligned}
v_{x}-i v_{y}= & V\left(1-\frac{R^{2}}{z^{2}}\right)+\frac{\Gamma}{2 \pi i}\left(-\frac{1}{z-z_{a}}+\frac{1}{z-z_{a}^{\prime}}\right. \\
& \left.+\frac{1}{z-z_{b}}-\frac{1}{z-z_{b}^{\prime}}\right),
\end{aligned}
$$

where $z_{b}=x_{b}+i\left(-R+\delta_{b}\right)$ and $z_{b}^{\prime}=R^{2} / z_{b}^{*}$. The flow velocity at $z=-i R$ is

$$
v_{x}=2 V+\frac{\Gamma}{\pi}\left[\frac{\delta_{b}}{x_{b}^{2}+\delta_{b}^{2}}-\frac{2 R+\delta_{a}}{x_{a}^{2}+\left(2 R+\delta_{a}\right)^{2}}\right] .
$$

When $\delta_{b}$ is positive, the first term in the square bracket of Eq. (16), i.e., the vortex at $z=z_{b}$, enhances the flow velocity. The vortex released from $z \simeq-i R$ therefore induces the creation of the subsequent vortex at $z \simeq-i R$. The velocity field in Eq. (15) for $x_{a}=4 R, \delta_{a}=0, x_{b}=R$, and $\delta_{b}=\xi$ is shown in Fig. 4(d), which well reproduces Fig. 2(d). Thus, vortices shed behind an obstacle induce the creation of an additional vortex, resulting in periodic vortex shedding, and ultimately hysteretic mechanism that allows this behavior to continue below the critical velocity $V_{c 1}$.

Figure 5 shows the radius $R$ and the velocity $V$ dependence of the flow patterns. The vortex shedding always occurs in the "vortex shedding" region and vortices are never created in the "no vortex" region. The "bistability" region lies between these two regions, in which a stationary laminar flow is stable but periodic vortex shedding is kept once it starts. For $R \lesssim 2 \xi$, the bistability region disappears, probably because $\delta_{b} \sim R$ in Eq. (16) is small and hence the enhancement of the successive vortex creation is less effective. Although the bistability region may also exist for $R \gtrsim 10 \xi$, it is difficult to determine the precise value of $V_{c 2}$ numerically, since the vortex shedding dynamics are aperiodic for large $R$, and are dependent on infinitesimal numerical noises.

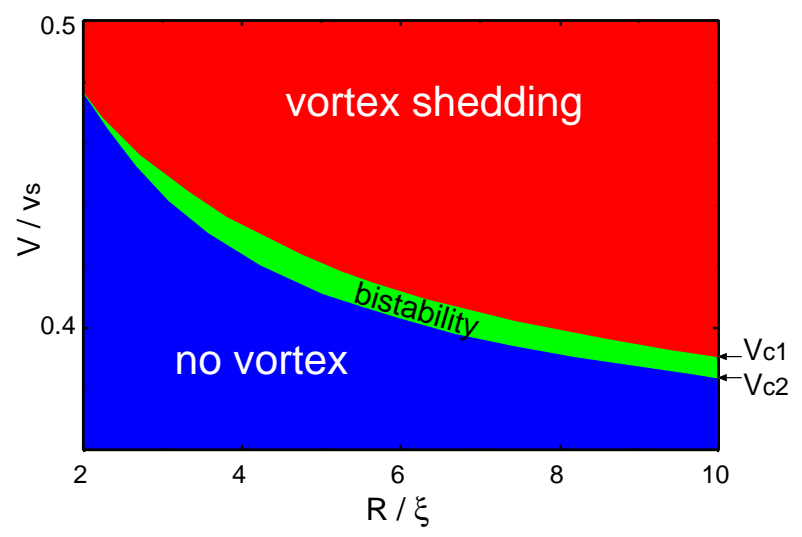

FIG. 5: (color online) Parameter diagram with respect to the obstacle radius $R$ and the velocity $V$. The vortex shedding always occurs in the "vortex shedding" region, no vortices are created in the "no vortex" region, and hysteresis appears in the "bistability" region. The boundaries between the bistability and vortex-shedding regions and the bistability and no-vortex regions are $V_{c 1}$ and $V_{c 2}$, respectively.

\section{B. Three dimensional system}

Next we examine a 3D system. We use a Gaussian potential for the moving obstacle as

$$
u(\boldsymbol{r})=100 \mu e^{-0.15\left(x^{2}+y^{2}+z^{2}\right) / \xi^{2}} .
$$

A spherical rigid potential analogous to that in Eq. (10) gives similar results. We prepare the initial state of a stationary laminar flow with $V=0.6 v_{s}$, which is below the critical velocity $V_{1 c} \simeq 0.601$ for vortex creation. In order to trigger the vortex shedding, an additional potential

$$
u_{\text {add }}(\boldsymbol{r})=0.2 \mu e^{-0.6\left[x^{2}+(y-d)^{2}+z^{2}\right] / \xi^{2}}
$$

is applied during $400 \tau<t<420 \tau$, where $d=6.3 \xi$. This additional potential is located at the edge of the potential $u$ in Eq. (17), triggering the generation of a quantized vortex ring, as shown in Fig. 6(b). Subsequent vortex creation is induced and periodic vortex shedding begins, as shown in Figs. 6(c) and 6(d), respectively. This result indicates that the bistability between the stationary laminar flow and periodic vortex shedding also exists in a $3 \mathrm{D}$ system.

We note that the vortex rings in a $3 \mathrm{D}$ system are topologically different from the vortex pairs in a $2 \mathrm{D}$ system. A vortex-antivortex pair in a $2 \mathrm{D}$ system corresponds to a vortex ring in a 3D system, since they propagate without changing their shapes. By contrast, a vortex-vortex pair in a 2D system, such as that seen in Fig. 2(e), has no counterpart in a $3 \mathrm{D}$ system, since two vortices rotate around one another for a vortex-vortex pair. Such rotation would tangle vortex rings in a 3D system. It is interesting that both 2D and 3D systems exhibit bistability despite the topological difference. 


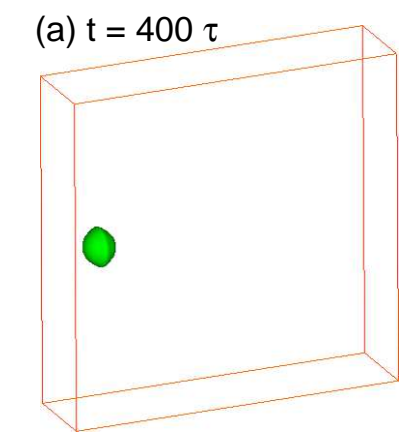

(b) $t=500 \tau$

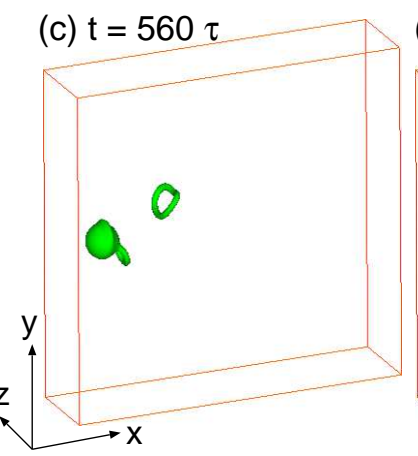

(d) $t=780 \tau$

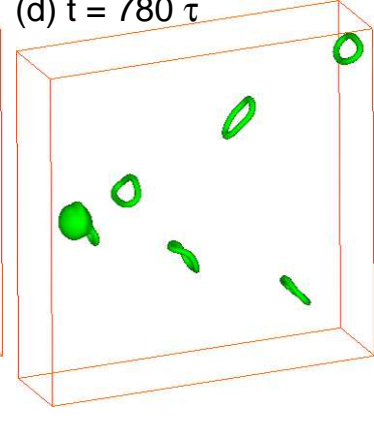

FIG. 6: (color online) Isodensity surfaces for the 3D dynamics. The Gaussian potential given by Eq. (17) moves in the $-x$ direction at a velocity $V=0.6 v_{s}$; the dynamics are given in the frame of reference of the potential. An additional potential given by Eq. (18) is applied during $400 \tau<t<420 \tau$. The size of the cuboidal frame is $126.4 \xi \times 126.4 \xi \times 31.6 \xi$. See the Supplemental Material for a movie of the dynamics.

[1] J. Zhang, S. Childress, A. Libchaber, and M. Shelley, Nature (London) 408, 835 (2000).

[2] M. Shelley, N. Vandenberghe, and J. Zhang, Phys. Rev. Lett. 94, 094302 (2005).

[3] R. E. D. Bishop and A. Y. Hassan, Proc. Roy. Soc. Lond. A 277, 51 (1964).

[4] M. M. Zdravkovich, J. Fluids Eng. 99, 618 (1977).

[5] C. H. K. Williamson, Annu. Rev. Fluid Mech. 28, 477 (1996).

[6] V. K. Horváth, J. R. Cressman, W. I. Goldburg, and X. L. Wu, Phys. Rev. E 61, R4702 (2000).

[7] T. B. Benjamin, Prod. R. Soc. Lond. A 359, 1 (1978); ibid. 359, 27 (1978).

[8] H. Kojima, W. Veith, S. J. Putterman, E. Guyon, and I. Rudnick, Phys. Rev. Lett. 27, 714 (1971).

[9] S. Eckel, J. G. Lee, F. Jendrzejewski, N. Murray, C. W. Clark, C. J. Lobb, W. D. Phillips, M. Edwards, and G. K. Campbell, Nature (London) 506, 200 (2014).

[10] C. Raman, M. Köhl, R. Onofrio, D. S. Durfee, C. E. Kuklewicz, Z. Hadzibabic, and W. Ketterle, Phys. Rev. Lett. 83, 2502 (1999).

[11] R. Onofrio, C. Raman, J. M. Vogels, J. R. Abo-Shaeer, A. P. Chikkatur, and W. Ketterle, Phys. Rev. Lett. 85, 2228 (2000).

[12] T. W. Neely, E. C. Samson, A. S. Bradley, M. J. Davis, and B. P. Anderson, Phys. Rev. Lett. 104, 160401 (2010).

[13] T. Frisch, Y. Pomeau, and S. Rica, Phys. Rev. Lett. 69,

\section{CONCLUSIONS}

We investigated the dynamics of a BEC with a moving obstacle potential, and found bistability between stationary laminar flow and periodic vortex shedding. When the velocity of the obstacle is gradually increased, quantized vortex shedding starts at the critical velocity $V_{c 1}$. On the other hand, when the velocity is gradually decreased from above $V_{c 1}$, the vortex shedding stops at a velocity $V_{c 2}$. We found that $V_{c 1}>V_{c 2}$ for an appropriately sized obstacle potential (Fig. 3). For a velocity $V_{c 1}>V>V_{c 2}$, a stationary laminar flow is stable, but periodic vortex shedding is maintained once it starts (Figs. 1 and 2). Such hysteretic behavior originates from the fact that the vortices released behind the obstacle enhance the velocity field around the obstacle, inducing subsequent vortex generation (Fig. (4). The bistability between the stationary laminar flow and periodic vortex shedding exists not only in 2D systems but also in 3D systems (Fig. 6).

\section{Acknowledgments}

We thank T. Kishimoto for fruitful discussions. This work was supported by a Grant-in-Aid for Scientific Research (No. 26400414) and a Grant-in-Aid for Scientific Research on Innovative Areas "Fluctuation \& Structure" (No. 25103007) from the Ministry of Education, Culture, Sports, Science and Technology of Japan.

1644 (1992).

[14] C. Nore, M. E. Brachet, and S. Fauve, Physica D 65, 154 (1993).

[15] B. Jackson, J. F. McCann, and C. S. Adams, Phys. Rev. Lett. 80, 3903 (1998).

[16] C. Josserand, Y. Pomeau, and S. Rica, Physica D 134, 111 (1999).

[17] C. Huepe and M. E. Brachet, Physica D 140, 126 (2000).

[18] J. S. Stießberger and W. Zwerger, Phys. Rev. A 62, 061601(R) (2000).

[19] C. Nore, C. Huepe, and M. E. Brachet, Phys. Rev. Lett. 84, 2191 (2000).

[20] T. Winiecki, B. Jackson, J. F. McCann, and C. S. Adams, J. Phys. B 33, 4069 (2000).

[21] S. Rica, Physica D 148, 221 (2001).

[22] A. Aftalion, Q. Du, and Y. Pomeau, Phys. Rev. Lett. 91, 090407 (2003).

[23] K. Sasaki, N. Suzuki, and H. Saito, Phys. Rev. Lett. 104, 150404 (2010).

[24] T. Aioi, T. Kadokura, T. Kishimoto, and H. Saito, Phys. Rev. X 1, 021003 (2011).

[25] H. Saito, T. Aioi, and T. Kadokura, Phys. Rev. B 86, 014504 (2012).

[26] F. Pinsker and N. G. Berloff, arXiv:1401.1517.

[27] G. W. Stagg, N. G. Parker, and C. F. Barenghi, arXiv:1401.4041

[28] W. H. Press, S. A. Teukolsky, W. T. Vetterling, B. P. 
Flannery, Numerical Recipes, 3rd ed, Sec. 20.7 (Cambridge Univ. Press, Cambridge, 2007).

[29] F. Dalfovo and S. Stringari, Phys. Rev. A 53, 2477 (1996).
[30] H. Lamb, Hydrodynamics, 6th ed, Sec. 155 (Dover, New York, 1945). 